\title{
Z-Spec: a broadband direct-detection millimeter-wave spectrometer -- instrument status and first results
}

L. Earle, P. Ade, J. Aguirre, R. Aikin, J. Battle, et al.

L. Earle, P. Ade, J. Aguirre, R. Aikin, J. Battle, J. Bock, C. M. Bradford, M. Dragovan, L. Duband, J. Glenn, G. Griffin, V. Hristov, P. Maloney, H. Matsuhara, B. Naylor, H. Nguyen, M. Yun, J. Zmuidzinas, "Z-Spec: a broadband direct-detection millimeter-wave spectrometer -- instrument status and first results," Proc. SPIE 6275, Millimeter and Submillimeter Detectors and Instrumentation for Astronomy III, 627510 (6 July 2006); doi:

10.1117/12.672309

SPIE Event: SPIE Astronomical Telescopes + Instrumentation, 2006, Orlando, Florida, United States 


\title{
Z-Spec: a broadband, direct-detection, millimeter-wave spectrometer - instrument status and first results
}

\author{
L. Earle $^{1 \dagger}$, P. Ade 2 , J. Aguirre 3,1 , R. Aikin ${ }^{1}$, J. Battle ${ }^{2}$, J. Bock ${ }^{2}$, C. M. Bradford 4 , \\ M. Dragovan 4 , L. Duband ${ }^{5}$, J. Glenn $^{1}$, G. Griffin ${ }^{6}$, V. Hristov ${ }^{6}$, P. Maloney ${ }^{1}$, H. Matsuhara ${ }^{7}$, \\ B. Naylor ${ }^{6}$, H. Nguyen ${ }^{4}$, M. Yun ${ }^{4}$, J. Zmuidzinas ${ }^{6}$ \\ ${ }^{1}$ Center for Astrophysics and Space Astronomy, University of Colorado, Boulder, CO \\ ${ }^{2}$ Cardiff University, Cardiff, UK \\ ${ }^{3}$ National Radio Astronomy Observatory \\ 4 Jet Propulsion Lab, Pasadena, CA \\ ${ }^{5}$ Commissariat à l'Energie Atomique, Grenoble, France \\ ${ }^{6}$ California Institute of Technology, Pasadena, CA \\ ${ }^{7}$ Institute of Space and Aeronautical Science, Sagamihara, Japan
}

\begin{abstract}
We report on the status of Z-Spec, including preliminary results of our first astronomical measurements. Z-Spec is a cryogenic, broadband, millimeter-wave grating spectrometer designed for molecular line surveys of galaxies, including carbon monoxide redshift measurements of high-redshift submillimeter sources. With an instantaneous bandwidth of 185-305 GHz, Z-Spec covers the entire $1 \mathrm{~mm}$ atmospheric transmission window with a resolving power of 200-400. The spectrometer employs the Waveguide Far-Infrared Spectrometer (WaFIRS) architecture, in which the light propagation is confined within a parallel-plate waveguide, resulting in a minimum mechanical envelope. Its array of 160 silicon-nitride micromesh bolometers is cooled to below $100 \mathrm{mK}$ for background-limited performance. With its sensitivity, broad bandwidth, and compactness, Z-Spec serves as a prototype for a future far-IR spectrometer aboard a cold telescope in space. Z-Spec successfully demonstrated functionality with a partial array of detectors and warm electronics during a week-long engineering run at the Caltech Submillimeter Observatory in June, 2005. We describe the instrument performance evaluated at the telescope and in subsequent laboratory tests and compare these results with design specifications. Following several modifications we returned to the telescope in April, 2006. We present a preliminary astronomical spectrum and discuss our plans to improve sensitivity and throughput to achieve our ultimate science goals.
\end{abstract}

Keywords: diffraction grating, millimeter-wave, waveguide, spectroscopy, bolometers, cryogenic, redshift

\section{INTRODUCTION}

Recent advancements in long-wavelength continuum instrumentation have enabled substantial progress toward resolving the far-infrared extragalactic background initially discovered by COBE in the $1990 \mathrm{~s} .{ }^{1}$ Deep observations by ground-based, large bolometer array cameras such as SCUBA, ${ }^{2}$ MAMBO, ${ }^{3}$ and Bolocam ${ }^{4}$ have detected numerous millimeter- and submillimeter-bright sources at moderate to high redshift. These sources are heavily dust-enshrouded and likely have high star-formation rates. At slightly higher frequencies, new surveys from Spitzer MIPS are producing numerous detections of luminous dusty galaxies, particularly at $24 \mu \mathrm{m} .^{5}$ This inventory will continue to grow as the next generation of imagers such as SCUBA-2 and new space missions such as Herschel Space Observatory come online. The next step is to examine what powers these galaxies and to interpret the source counts in a framework of galaxy formation and evolution through detailed studies of interstellar medium gas conditions and redshift measurements. However, many of these galaxies have no observable optical counterparts due to dust obscuration and positional uncertainty at these low frequencies; consequently our current understanding suffers from limitations in follow-up techniques and a dearth of spectroscopic data.

\footnotetext{
$\dagger$ For further information contact Lieko Earle: lieko@casa.colorado.edu or (303)492-4349
} 
Challenges notwithstanding, recent years have seen intriguing breakthroughs. For example, redshifts have successfully been measured for a subsample of submillimeter galaxies with the Keck LRIS spectrograph, revealing a median $\overline{\mathrm{Z}}$ of 2.4. ${ }^{6}$ Follow-up observations from Plateau de Bure (PdBI) have enabled millimeter-wave CO detections for a handful of these sources with redshifts. ${ }^{7}$ These results, while exciting, are subject to the caveat that they rely first on accurate positions determined from VLA radio continuum images, likely biasing the sample in favor of sources with $\mathrm{z} \lesssim 3$.

Clearly, sensitive spectroscopy at wavelengths immune to dust-extinction is the ideal approach to further investigation of the continuum discoveries. To this end, a natural choice for ground-based observations is at 1 $\mathrm{mm}$, where the atmospheric transmission window is reliable and broadband. To address this need, we have built Z-Spec, a broadband, millimeter-wave, diffraction grating spectrometer. Its sensitive detector array and instantaneous bandwidth of 185-305 GHz opens the door to spectroscopic survey capabilities previously unrealized. Our primary science objectives are to:

1. Measure redshifts of submillimeter galaxies using CO lines - Carbon monoxide is the second most abundant molecule in the ISM after $\mathrm{H}_{2}$ and is the dominant coolant. Its ladder of rotational transitions is spaced every $115 \mathrm{GHz}$ in the rest frame. When two or more CO lines are simultaneously shifted into Z-Spec's bandpass, as is the case for sources at $\mathrm{z} \gtrsim 0.9$, the redshift can be uniquely determined from the ratio of the line frequencies. The advantage to this method of measuring $\mathrm{z}$ is that it does not rely on detection at other frequencies, thus reducing selection effects.

2. Conduct systematic line surveys of local galaxies - The ultraluminous and luminous infrared galaxies (ULIRGs and LIRGs) first revealed in the 1980s by IRAS exhibit characteristics similar to those of high-z submillimeter galaxies responsible for the far-IR background: They are bright in carbon monoxide, indicating large molecular gas reservoirs (necessary for star-formation), and are heavily dustenshrouded, emitting primarily in the far-IR. As such, they provide an excellent proxy for investigating gas conditions in high-z galaxies. The 1 $\mathrm{mm}$ window is rich with molecular rotational transitions; notable tracers available in Z-Spec's bandpass are: CO isotopes $\mathrm{J}=2 \rightarrow 1$ (optically thick ${ }^{12} \mathrm{CO}$ for temperatures, optically thin ${ }^{13} \mathrm{CO}$ and $\mathrm{C}^{18} \mathrm{O}$ for mass), $\mathrm{HCN} \mathrm{J}=3 \rightarrow 2$ and $\mathrm{HNC} \mathrm{J}=3 \rightarrow 2$ (for density), and $\mathrm{CN} \mathrm{J}=2 \rightarrow 1$ (for dense, photodissociated regions). These spectra will complement existing data from lower-J transitions of the same species previously obtained at radio frequencies. ${ }^{8}$

Z-Spec serves as a millimeter-wave testbed for the WaFIRS spectrometer concept, which combines a parallel-plate waveguide with a diffraction grating, and whose design is scalable to higher frequencies in the far-IR. Compact in size and superior in sensitivity, WaFIRS is a suitable candidate for spectroscopy from a future cold space-borne platform (e.g. BLISS on SPICA). ${ }^{9,10}$ We present an overview of ZSpec and its status report, including preliminary data from our first astronomical measurements. Further instrument characterization and

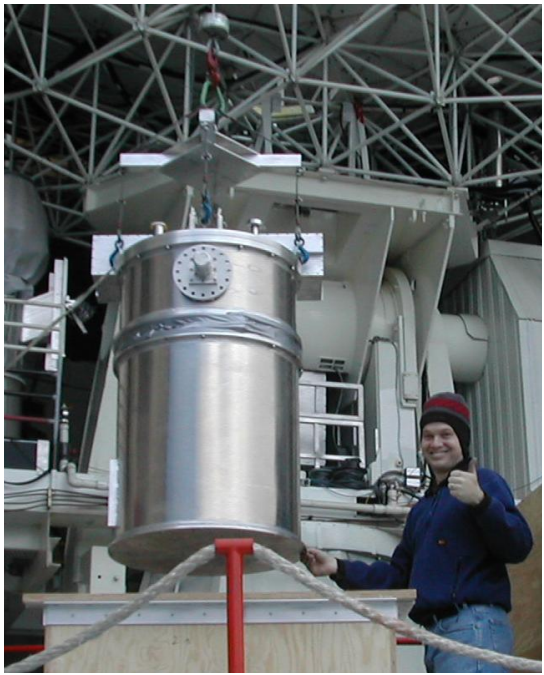

Figure 1. The Z-Spec cryostat upon arrival at the Caltech Submillimeter Observatory. Caltech graduate student Bret Naylor is shown for scale. modifications are currently underway to achieve the designed performance specifications.

\section{INSTRUMENT DESCRIPTION}

An overview of the instrument design is presented here. The following previous SPIE proceedings should be consulted for detailed descriptions of subcomponents: WaFIRS concept in $2002 ;{ }^{11}$ instrument design, in particular, development of waveguide and coupling structures in $2002 ;^{12} \mathrm{Z}$-Spec's WaFIRS module details and results of first laboratory spectrometer testing in $2004 .{ }^{13}$ 


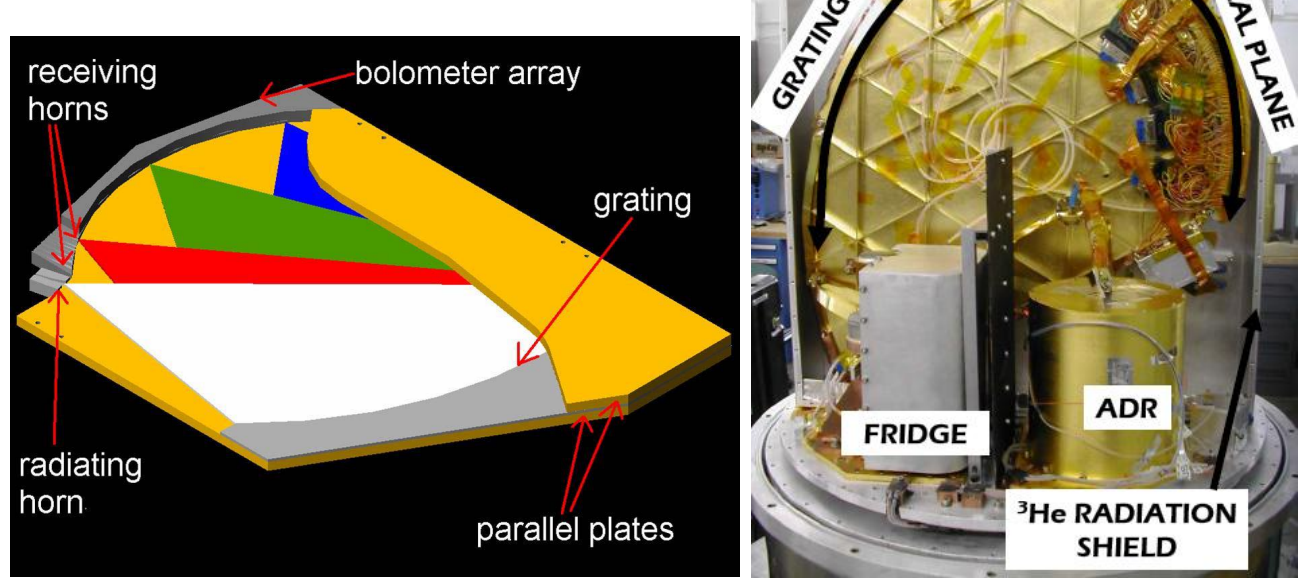

Figure 2. [Left] Schematic of WaFIRS with top plate cut away. The incoming light (shown in white) illuminates the curved grating surface, and is dispersed and focused (shown in 3 shades) along the focal plane opposite. The diffraction grating is based on a Rowland circle, and each facet is positioned to provide stigmatic performance at 2 frequencies. [Right] Photograph of Z-Spec's WaFIRS module inside the cryostat. It measures $62 \mathrm{~cm} \mathrm{x} 48 \mathrm{~cm} \times 3.3 \mathrm{~cm}$ and is machined of aluminum 6061-T6 and goldplated. The triangular cutouts provide structural rigidity as well as lightweighting.

The Waveguide Far-Infrared Spectrometer (WaFIRS) is an innovative technology which exploits the sensitivity and bandwidth advantages of a diffraction grating while significantly reducing overall volume by confining the light propagation between a pair of parallel conducting plates. A schematic and photo are shown in Figure 2. The basic design is based on the Rowland circle, where the arc of the grating simultaneously disperses and focuses the radiation. The grating facets are positioned to provide stigmatic performance at two frequencies. There are 480 facets, with a blaze angle of 27 degrees, and the resolving power decreases with frequency from 400 to 200 across the band. The waveguide plate separation is $2.5 \mathrm{~mm}$, sufficiently wide to minimize waveguide losses, yet narrow enough to limit exciting higher order modes. Z-Spec's WaFIRS module measures $62 \mathrm{~cm} \mathrm{x} 48$ $\mathrm{cm} \times 3.3 \mathrm{~cm}$.

A circular corrugated feedhorn matched to the telescope relay optics receives incoming radiation and couples it to the spectrometer via a tapered rectangular waveguide. The grating is slightly over-illuminated to optimize in favor of spectral resolution over total throughput. The diffracted light arrives at the focal arc where waveguide bends direct each frequency bin alternately above and below the plane of the spectrometer plates; Figure 3 illustrates the waveguide bendblocks. There are 8 bendblocks along the arc, each comprised of 20 waveguide channels. At the end of each waveguide bend is an individually mounted detector. This method eliminates the need for tapered feeds and allows for a close-packed array of detectors, minimizing losses.

The detectors are $160 \mathrm{Si}_{3} \mathrm{~N}_{4}$ micromesh bolometers with neutron-transmutation-doped (NTD) germanium thermistors. The bolometers are etched from silicon wafers $370 \mu \mathrm{m}$ thick and attached to deep-trenched etched silicon backshorts. To ensure good efficiency two different quarter-wave backshort distances are used for high and low frequency detectors. Each bolometer is mounted on an alumina "key" and screwed onto a waveguide bendblock channel. That the pixels are individually replaceable is vital; Z-Spec does not oversample spectrally, so effective spectroscopy relies on $100 \%$ detector yield. For our expected background loading at $1 \mathrm{~mm}$, the target thermal conductance is $15 \mathrm{pW} / \mathrm{K}$ at $100 \mathrm{mK}$, corresponding to dark NEPs of $\sim 4 \times 10^{-18} \mathrm{~W} / \sqrt{\mathrm{Hz}}$ with thermal time constants of $\sim 15 \mathrm{~ms}$.

To reject out-of-band radiation, low-pass metal mesh interference filters are placed along the beam path, at $77 \mathrm{~K}, 4.2 \mathrm{~K}, 350 \mathrm{mK}$, and on the feedhorn at $<100 \mathrm{mK}$. A $3 \mathrm{~mm}$-thick layer of Fluorogold is employed at $4.2 \mathrm{~K}$ as an additional thermal IR blocker. Lower frequencies are naturally eliminated by the waveguide cutoffs. 

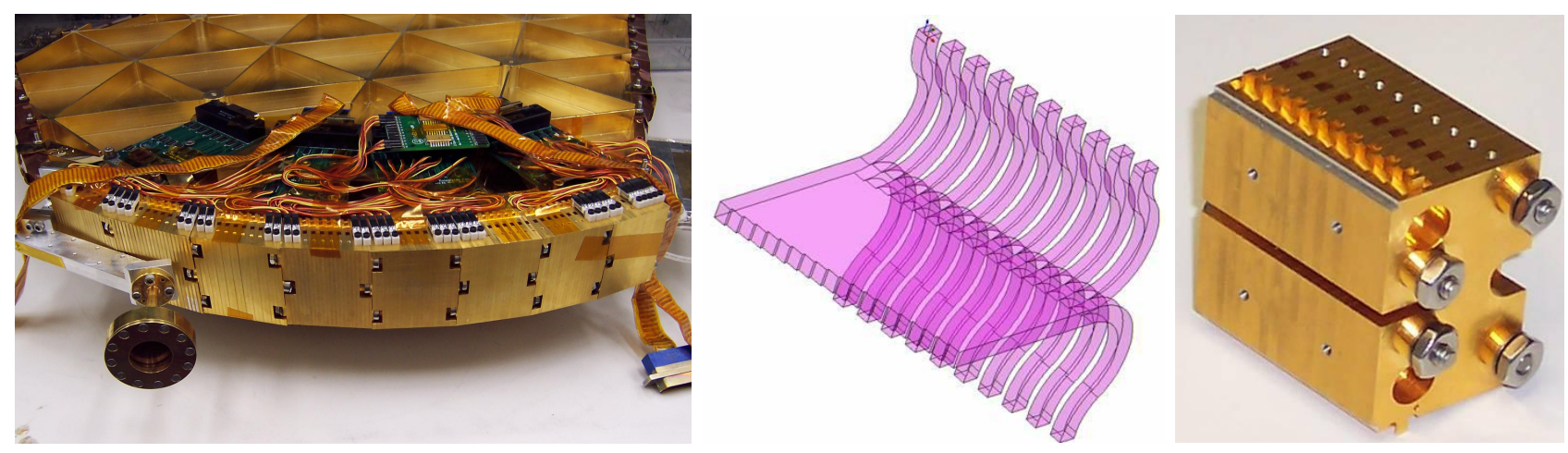

Figure 3. [Left] The input feedhorn and rectangular waveguide are positioned on the edge adjacent to the focal plane. Along the focal arc are 8 waveguide bendblocks, each consisting of 20 waveguide channels which couple the diffracted light to individual detectors. A few detectors are shown on the top side of the blocks. [Center] Schematic of curved waveguides inside each bendblock. [Right] View of an individual bendblock, in same orientation as schematic. The light comes in through the gap on the left and is directed alternately toward the top and bottom of block, where individual detectors are mounted.

The detectors, grating, parallel plates, bendblocks and input feedhorn total approximately $5 \mathrm{~kg}$, and this entire mass is cooled to below $100 \mathrm{mK}$ via 2 -stage cooling supplied by a ${ }^{3} \mathrm{He} /{ }^{4} \mathrm{He}$ closed-cycle fridge and an adiabatic demagnetization refrigerator (ADR). The utility of the helium fridge is two-fold: to shield the cold stage from 4 $\mathrm{K}$ radiation, and to pre-cool the spectrometer assembly to $\sim 450 \mathrm{mK}$. At these temperatures the entropy of the aluminum grating is small compared to the post-demagnetization entropy available in the ferric ammonium alum (FAA) salt pill, so cooling the entire grating plus detectors is efficient, with an ADR hold time of 16-20 hours. The system is enclosed in a standard liquid nitrogen / liquid helium cryostat, with helium consumption averaging $0.5 \mathrm{li} / \mathrm{hr}$ when running a fridge/ADR cycle daily. For the detectors to reach their operating temperature takes of order 3 days from initial pumpdown; we employ ${ }^{4} \mathrm{He}$ exchange gas to expedite the cooling to $77 \mathrm{~K}$.

The bolometers are AC-biased, and their high-impedance signal is read out via matched pairs of cryogenic JFETs before being brought out to the warm electronics. The $\mathrm{LN}_{2}$-cooled JFETs self-heat to $140 \mathrm{~K}$. The warm electronics consist of a low-pass filter and a preamplifier with a gain of 200 at DC. The signal from the preamp is passed to a DSP which may be operated in DC or AC mode. In DC mode, it simultaneously low-passes and digitizes the signal using a sigma-delta algorithm. In AC mode, it also demodulates the signal against the AC bias reference. Two channels of 24-bit data words at 22-bit resolution are produced: these are the in- and outof-phase demodulations in AC, while the second channel is redundant in DC mode. The final sample rate is half the bias frequency in AC mode, and can be set arbitrarily in DC. The DC mode is used for taking loadcurves, while the AC mode is used for science data acquisition. The noise of the electronics is $\sim 10-12 \mathrm{nV} / \sqrt{\mathrm{Hz}}$, with a $1 / \mathrm{f}$ knee at $<0.1 \mathrm{~Hz}$. The electronics readout is grouped into 8 boxes of 24 channels each, along with one box for the bias reference and JFET power, and another for housekeeping signals. All 10 boxes transmit UDP packets independently, which routed through a hub and are acquired and timestamped by a data acquisition computer.

At the CSO, Z-Spec shares the right Nasmyth port with SHARCII and other instruments which mount to its platform. An off-axis ellipsoid converts the $f / 12$ beam from the secondary to $f / 3$. To accommodate changing weather conditions, a fold mirror mounted on a linear bearing facilitates ease of switching observing modes between Z-Spec and another (higher-frequency) instrument such as SHARCII. The predicted efficiency is $89-91 \%$, limited by the edge taper on the secondary.

\section{INSTRUMENT CHARACTERIZATION}

The spectral resolution was determined using a Fourier Transform spectrometer with a resolution of $100 \mathrm{MHz}$. Figure 4 shows the spectra of 12 adjacent channels in the $250-260 \mathrm{GHz}$ range, near the center of Z-Spec's band. The peak height is normalized to unity to take out the channel-to-channel variation in detector response. The spectra are uniform in profile and centrally peaked. A polynomial fit to the measured center-band frequencies 


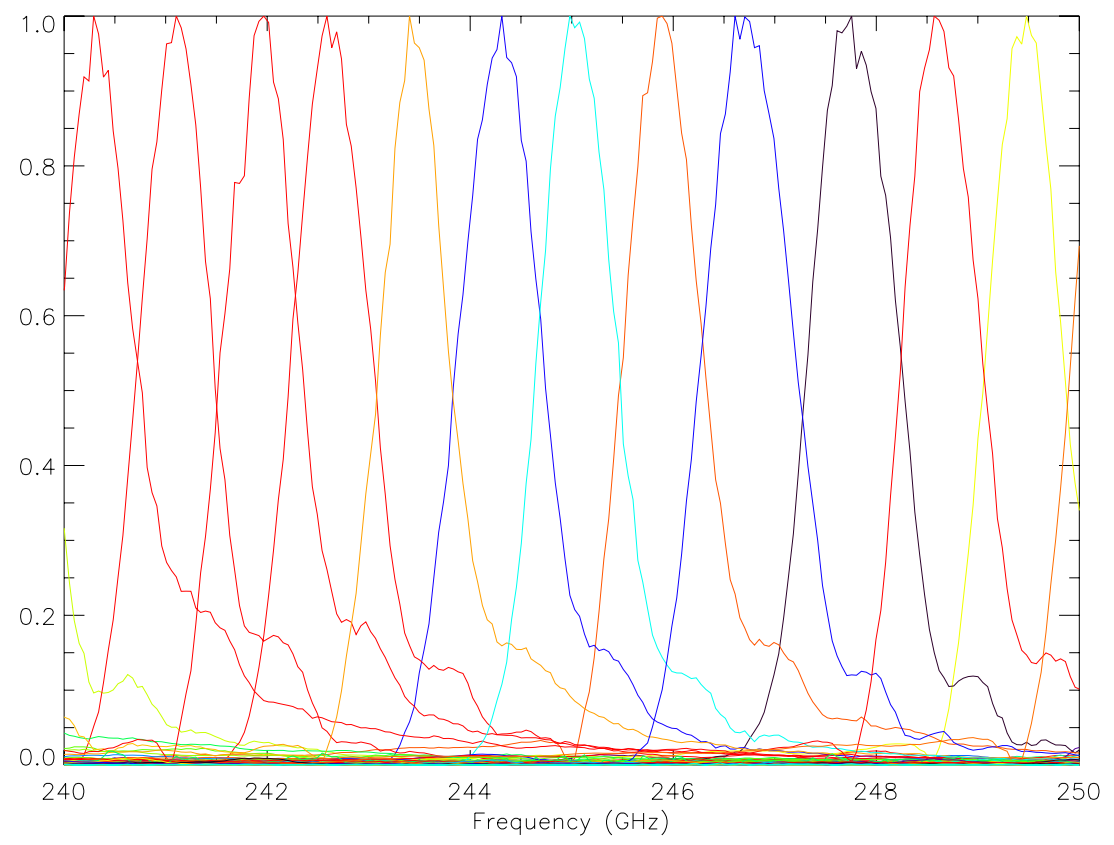

Figure 4. Laboratory FTS spectra of 12 adjacent channels demonstrating the well-shaped bandpasses and uniform channel-to-channel resolution. The peak height of each channel has been normalized to 1.

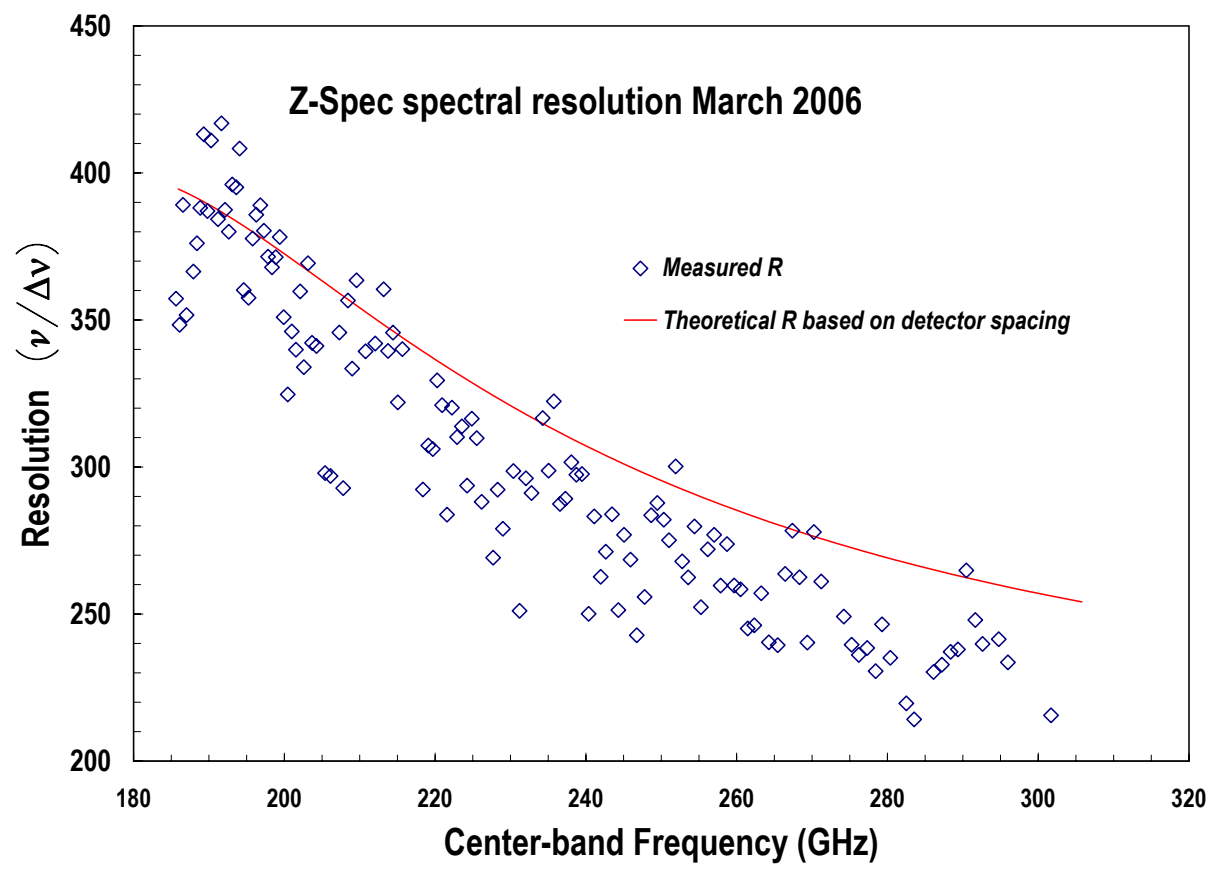

Figure 5. FTS-measured spectral resolution. R varies from 200 to 400 across the band. The theoretical curve shows expected $\mathrm{R}$ based on frequency spacing between adjacent channels. 


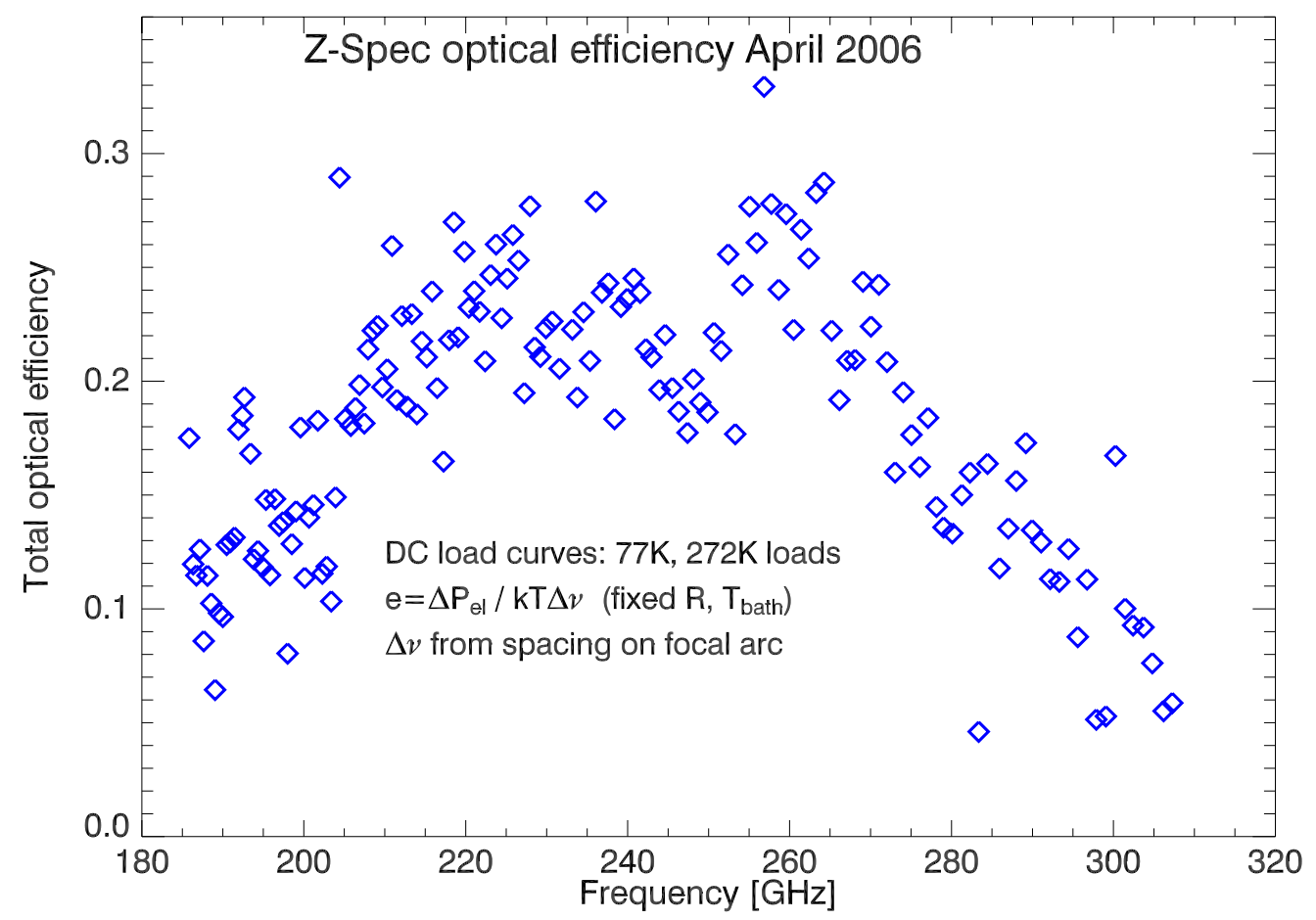

Figure 6. Z-Spec coupling efficiency based on ambient and $77 \mathrm{~K}$ load curves. The end-to-end throughput is $20 \sim 25 \%$ near the center of the band.

was used to generate a look-up table for each detector channel, and the results are in good agreement both with previous FTS measurements and with astronomical lines detected during our observations. The spectral resolution is computed using $\mathrm{R}=\nu / \Delta \nu$, where $\Delta \nu$ is taken to be the FWHM of the Gaussian fit to the measured spectra. The results are compared with a theoretical curve based on the detector spacing in Figure 5. The resolution varies from 200 to 400 across the band.

Figure 6 shows the end-to-end (dewar window $\rightarrow$ bolometer absorption) throughput. The efficiency is $20 \sim$ $25 \%$ near the band center, tapering down toward the band edges. The low-frequency channels may be suffering from waveguide losses, while the grating blaze efficiencies may be affecting the high-frequency end.

Several modifications to the instrument system subsequent to the engineering run contributed to improved performance at the telescope. We have essentially eliminated the microphonics by both implementing more careful tie-downs of all signal cables between the bolometers and JFETs, and by installing vibration isolation between the cryostat and its platform. In an effort to reduce excess loading, new blocking filters were installed at the $77 \mathrm{~K}$ window and the inside edges of the parallel-plate waveguide were coated with carbon-black. Detector yield on the focal plane is approaching $100 \%$, with 158 out of 160 channels currently in operation. Problems with the electronics firmware, which caused dropouts, bias problems, and communication difficulties have been fixed. The data acquisition software has been improved to avoid dropouts as well.

There is still evidence of excess photon noise within the cryostat higher than what we can tolerate in order to be limited by only the sky and telescope background. Z-Spec's sensitivity at the CSO computed from a $\tau_{225 G H z}=0.1$, airmass $=2$ observation is $1200 \mathrm{mJy} \sqrt{s}$, a factor of 2 higher than our design goals. After this measurement, we began pumping on both the helium and nitrogen baths during observations to compensate for the excess loading. 


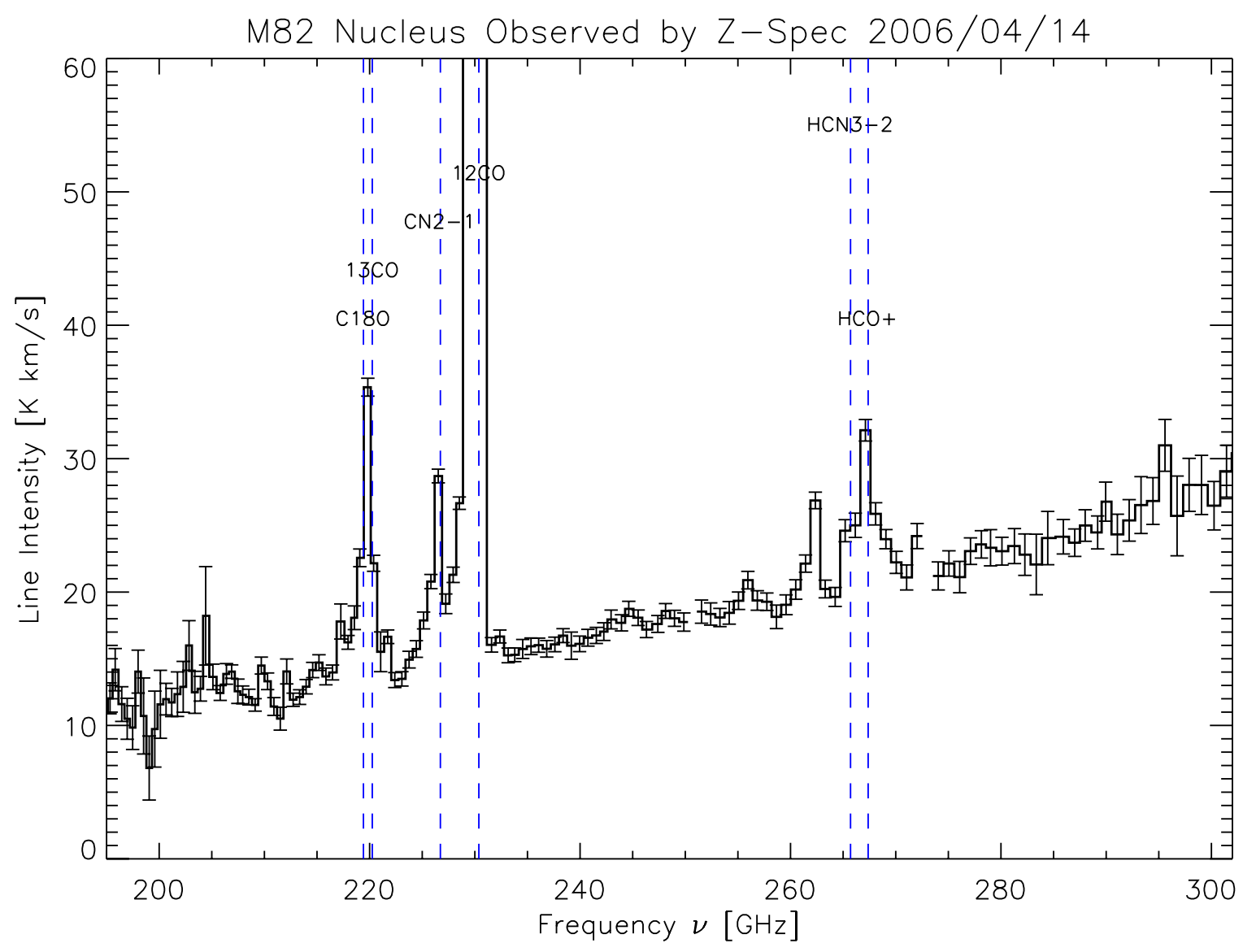

Figure 7. Z-Spec spectrum of the nucleus of M82, shown with 1-sigma error bars. In addition to several spectral lines, the dust continuum is clearly detected, with an upward slope toward high frequencies. This rise is due both to the increasing bandwidth and the steeper-than-thermal dust spectrum. M82 is extended; the spectrum shown here has not been corrected for the beam-filling factor.

\section{PRELIMINARY ASTRONOMICAL SPECTRUM}

Figure 7 shows a preliminary reduction of a spectrum of the nucleus of M82. M82 is the nearest starburst galaxy. The nuclear region is extended on the scale of Z-spec's beam; the spectrum in Figure 7 represents only one of several pointings that were taken. The chop throw was 120 " at $1 \mathrm{~Hz}$. Seven lines (some blended), including ${ }^{12} \mathrm{CO}$, were detected above $5 \sigma$ in 4800 seconds of integration; these lines are shown in Table 1. At Z-Spec's resolution, the $\mathrm{C}^{18} \mathrm{O}$ and ${ }^{13} \mathrm{CO}$ are unresolved from one another. The continuum is also clearly detected. The ability of Z-spec to detect multiple lines and continuum in a single observation and with a single calibration allows accurate determinations of the line-to-continuum and line-to-line ratios. The dust spectral index at $\sim 250$ GHz may also be determined from the slope of the spectrum.

\section{FURTHER INSTRUMENT OPTIMIZATION}

Further laboratory testing and modifications are ongoing in preparation for Z-Spec's next observing run in late 2006 or early 2007. Z-Spec has demonstrated functionality as a moderate-resolution, broadband spectrometer. Spectra of nearby galaxies and molecular clouds have been obtained and analysis for these data are underway. The remaining issue to address is that of instrument sensitivity, which must be improved for observations of high-redshift submillimeter galaxies from the CSO. 


\begin{tabular}{|c|c|c|c|c|}
\hline Species & Transition & $\begin{array}{c}\nu_{\text {rest }} \\
G H z\end{array}$ & $\begin{array}{c}\text { Intensity } \\
K k m / s\end{array}$ & $\begin{array}{c}\text { Beam Size } \\
\text { arcsec }\end{array}$ \\
\hline $\mathrm{C}^{18} \mathrm{O}$ & $2 \rightarrow 1$ & 219.56 & 44 & 32.5 \\
${ }^{13} \mathrm{CO}$ & $2 \rightarrow 1$ & 220.40 & & \\
\hline $\mathrm{CN}$ & $2 \rightarrow 1$ & 226.88 & 29 & 31.5 \\
\hline $\mathrm{CO}$ & $2 \rightarrow 1$ & 230.54 & 460 & 31 \\
\hline $\mathrm{HCN}$ & $3 \rightarrow 2$ & 265.89 & 9 & 27 \\
\hline $\mathrm{HCO}^{+}$ & $3 \rightarrow 2$ & 267.56 & 18 & 27 \\
\hline
\end{tabular}

Table 1. Z-Spec line detections in M82 nucleus.

In addition to studying the filter stack to determine whether the mesh layers could be the cause of scattering inside the dewar, we are investigating the possibility of installing cryogenic baffles. Beam maps in the laboratory will help to understand whether the coupling is adequate: Load curves taken with a cold load at the telescope's elevation bearing (between relay optics M3 and M4) along with data from coupling to Jupiter indicate spillover losses along the optical path. Crude measurements of the secondary edge taper were made by holding a warm load at the edges of the mirror, and analysis of this data is pending to determine whether the bulk of the spillover occurs at the secondary.

\section{CONCLUSION}

We present results of laboratory tests and preliminary astronomical data obtained with Z-Spec, a cryogenic broadband millimeter-wave spectrometer which employs a waveguide-coupled curved diffraction grating. FTS measurements in the laboratory confirm an instantaneous bandwidth of 185-305 GHz with a spectral resolution which varies from 200 to 400 across the band. The end-to-end throughput of the instrument is $20-25 \%$ in the band center. We have begun astronomical observations with Z-Spec at the Caltech Submillimeter Observatory. Z-Spec demonstrates the viability of the WaFIRS concept for future space-borne spectroscopy in the far-IR. Further instrument characterization in the laboratory is currently underway in anticipation of returning to the telescope this winter with improved performance.

\section{ACKNOWLEDGEMENTS}

We thank the entire CSO staff for their invaluable support as we launch this new instrument. L. Earle is supported by a NASA GSRP fellowship (NGTS-50478). J. Aguirre is funded by a Jansky Fellowship from NRAO. J. Glenn acknowledges an NSF Career Grant in support of Z-Spec (AST-0239270) and an Innovation Award from the Research Corporation (RI0928). This work was supported in part by NASA SARA grants NAGS-11911 and NAGS-12788.

\section{REFERENCES}

1. Fixsen, D.J. et al., "The Spectrum of the extragalactic far-infrared background from the COBE FIRAS observations," AJ, 508:123, 1998.

2. Holland, W.S. et al., "SCUBA: a common-user submillimetre camera operating at the James Clerk Maxwell Telescope," Monthly Notices of the RAS, 303:659, 1999.

3. Bertoldi, F. et al., "Three high-redshift millimeter sources and their radio and near-infrared identifications," $A \& A, 360: 92,2000$.

4. Laurent, G. et al., "The Bolocam Lockman Hole Millimeter-Wave Galaxy Survey: Galaxy Candidates and Number Counts," ApJ, 623:742, 2005.

5. Papovich, C. et al., "The 24 Micron Source Counts in Deep Spitzer Space Telescope Surveys," ApJS, 154:70, 2004. 
6. Chapman, S. C. et al., "A Redshift Survey of the Submillimeter Galaxy Population," ApJ, 622:722, 2005.

7. Greve, T. R. et al., "An interferometric CO survey of luminous submillimetre galaxies," MNRAS, 359:1165, 2005.

8. Aalto, S. et al., "CN and HNC line emission in IR luminous galaxies," A\&A, 381:783, 2002.

9. Bradford, C. M. et al., "BLISS and SPICA: Revealing the History of Energy Production in Dusty Galaxies with Far-IR Spectroscopy at the Background Limit," American Astronomical Society Meeting 205, 205:10, 2004.

10. Bradford, C. M. et al., "BLISS for SPICA: far-infrared spectroscopy at the background limit," Proc. SPIE, 6265-115, 2006.

11. Bradford, C. M. et al., "WaFIRS, a waveguide far-IR spectrometer for spaceborne astrophysics," Proc. SPIE, 4850:162, 2002.

12. Naylor, B. et al., "Z-Spec: a broadband, direct-detection, millimeter-wave spectrometer," Proc. SPIE, 4855:239, 2002.

13. Bradford, C. M. et al., "Z-Spec: a broadband millimeter-wave grating spectrometer - design, construction, and first cryogenic measurements," Proc. SPIE, 5498:257, 2004. 